\title{
Micro-Ribonucleic Acid Profiles From Microarray in Ankylosing Spondylitis
}

\author{
Hye-Mi JIN, ${ }^{1}$ Young-Nan CHO, ${ }^{1}$ Seung-Jung KEE, ${ }^{2}$ Shin-Seok LEE, ${ }^{1}$ Yong-Wook PARK, ${ }^{1}$ Tae-Jong KIM ${ }^{1}$ \\ ${ }^{1}$ Department of Rheumatology, Research Institute of Medical Sciences, \\ Chonnam National University Medical School and Hospital, Gwangju, South Korea \\ ${ }^{2}$ Department of Laboratory Medicine, Research Institute of Medical Sciences, \\ Chonnam National University Medical School and Hospital, Gwangju, South Korea
}

\begin{abstract}
Objectives: This study aims to detect candidate micro-ribonucleic acids (miRNAs) from microarray within peripheral blood mononuclear cells and synovial fluid mononuclear cells in patients with ankylosing spondylitis (AS).

Patients and methods: Samples from three AS patients ( 3 males, mean age $37.3 \pm 2.5$ years; range 35 to 40 years) and three healthy controls ( 3 males, mean age $39.0 \pm 2.6$ years; range 37 to 42 years) were obtained for miRNA microarray. The microarray experiment proceeded only when the quality of total RNAs were considered to have "passed", and their integrity was good by total RNA quality control using Agilent Bioanalyzer 2100. Hierarchical clustering was performed to understand the impact of the storage condition on the miRNA expression profiles. MiScript primer assays were used for semiquantitative determination of the expression of human miRNAs to validate results from miRNA microarray.

Results: A total of 887 miRNAs were screened by microarray among groups. After normalization of the raw data, we noted that the expression of five miRNAs was significantly lower (fold change $\leq 0.5$ and $p \leq 0.05$ ) and only hsa-miR-424-5p was significantly higher in AS peripheral blood mononuclear cell (fold change $\geq 2$ and $p \leq 0.05$ ). In AS synovial fluid mononuclear cells, we identified that expressions of 16 miRNAs were significantly down regulated whereas only hsa-miR-424-5p was significantly upregulated (fold change $\geq 2$ and $p \leq 0.05$ ). All above-mentioned miRNAs were reevaluated for further validation. Finally, significantly increased hsa-miR-424-5p and decreased hsa-miR-377 were found in synovial fluid mononuclear cells from AS patients compared with healthy controls. Based on target prediction programs and published papers, potential target genes and its pathways were screened.
\end{abstract}

Conclusion: miR-424-5p was increased and miR-377 was decreased in synovial fluid mononuclear cells from patients with AS. These two miRs might have functional roles in patients with arthritis via different pathways.

Keywords: Ankylosing spondylitis; microarray analysis; micro-ribonucleic acid.

Ankylosing spondylitis (AS) is characterized by inflammation of the axial skeleton and sacroiliac joints. Peripheral joints and certain extra-articular organs, including the eyes, skin, and cardiovascular system, are affected to a lesser degree. ${ }^{1}$ Peripheral joint involvement is a presenting feature in only $10-20 \%$ of patients, and occurs during the disease course in 30-40\% of patients. ${ }^{2,3}$ Disease activity is higher in patients with peripheral joint disease, when compared with patients where the disease is limited to the axial skeleton. ${ }^{4}$
Micro-ribonucleic acids (miRNAs) are noncoding RNA oligonucleotides that have been highly conserved during evolution and have recently emerged as potent regulators of gene expression of posttranscriptional regulators. ${ }^{5}$ miRNAs have been identified and appear to be critical for finetuning many biological processes, in addition to offering the prospect of multiple targets. ${ }^{6,7}$ Emerging data suggests that single miRNA species can profoundly alter the phenotype and outcome of immune responses. . $^{8-10}$ Although dysregulated expression profiles of miRNAs have 
been identified in the blood of patients with immune disease, ${ }^{11}$ only limited data is available regarding miRNA with regard to AS. ${ }^{12-14}$

Therefore, in this study, we aimed to detect candidate miRNAs from microarray within peripheral blood mononuclear cells (PBMCs) and synovial fluid mononuclear cells (SFMCs) in patients with AS.

\section{PATIENTS AND METHODS}

Samples from three AS patients ( 3 males, mean age $37.3 \pm 2.5$ years; range 35 to 40 years) with peripheral arthritis and three healthy controls (HCs) (3 males, mean age 39.0 \pm 2.6 years; range 37 to 42 years) were obtained between December 2011 and January 2012 from Chonnam National University Hospital for miRNA microarray. To validate miRNA expression, samples from 25 $\mathrm{HCs}$, and paired peripheral blood and synovial fluid from 21 patients with AS were obtained. The study was approved by the Institutional Review Board of Chonnam National University Hospital, and written informed consent was obtained from all participants.

Peripheral venous blood and synovial fluid samples were collected in heparin-containing tubes. PBMCs and SFMCs were isolated by density-gradient centrifugation using FicollPaque Plus solution (Amersham Biosciences, Uppsala, Sweden). Freshly isolated PBMCs were suspended in a complete medium, consisting of RPMI 1640, 2 mM L-glutamine, 100 units $/ \mathrm{mL}$ of penicillin, and $100 \mu \mathrm{g} / \mathrm{mL}$ of streptomycin, supplemented with 10\% FBS (Gibco BRL, Grand Island, NY, USA).

Total RNA was isolated using the miRNeasy kit (Qiagen Inc., Valencia, CA, USA). The presence of miRNA in the total RNA was assayed using the Agilent 2100 Bioanalyzer Small RNA assay (Agilent Technologies, CA, USA). The microarray experiment proceeded only when the quality of total RNAs were considered as "passed" and their integrity was good by total RNA quality control using Agilent Bioanalyzer 2100. 100 ng of each RNA sample were hybridized to Agilent Human microRNA Microarray v2.0 (G4470B, Agilent Technologies). MicroRNA labeling, hybridization, and washing were carried out following Agilent's instructions. Images of hybridized microarrays were acquired with a microarray scanner (Agilent G2565BA), and features were extracted using the agilent feature extraction image analysis tool version A.9.5.3.1. To understand the impact of the storage condition on the miRNA expression profiles, hierarchical clustering was performed. Microarray data were quantile-normalized and analyzed using GeneSpring software (Agilent).

The miScript Reverse Transcription Kit (Qiagen $\mathrm{GmbH}$, Hilden, Germany) was used for complementary deoxyribonucleic acid (cDNA) preparation. MiScript primer assays (Qiagen $\mathrm{GmbH}$, Hilden, Germany) were used for semiquantitative determination of the expression of human miRNAs. The expression of U6B snRNA was used as an endogenous control. A threshold cycle $(\mathrm{CT})$ was observed in the exponential phase of amplification, and quantification of relative expression levels were performed using standard curves for target genes and the endogenous control. Geometric means were used to calculate the $\Delta \Delta \mathrm{CT}$ (delta-delta $\mathrm{CT}$ ) values and were expressed as 2- $\Delta \Delta \mathrm{CT}$. The value of each control sample was set at one and used to calculate the fold-change of the target genes.

\section{Statistical analysis}

The Kruskal-Wallis test was used for the comparison of gene expression among groups. Dunn's test was used post-hoc to compare all pairs of groups. A p-value $<0.05$ was considered statistically significant. All statistical analyses were performed using PASW version 17.0 software (SPSS Inc., Chicago, IL, USA).

\section{RESULTS}

A total of 887 miRNAs were screened by microarray among groups (Figure 1). After normalization of the raw data, we noted that the expression of five miRNAs, including hsa-miR-136, hsa-miR-29c*, hsa-miR-376a, hsamiR-376c, hsa-miR-377, were significantly lower (fold change $\leq 0.5$ and $p \leq 0.05$ ) and only hsa-miR424-5p was significantly higher in AS PBMCs (fold change $\geq 2$ and $p \leq 0.05$, Table 1). In AS SFMCs, it was identified that expressions of 16 miRNAs were significantly down regulated: hsa-miR-125b, hsa-miR-126, hsa-miR-126*, hsa-miR-136, hsa-miR-144, hsa-miR-151-3p, 
hsa-miR-199a-5p, hsa-miR-29c*, hsa-miR-30b, hsa-miR-326, hsa-miR-376a, hsa-miR-376c, hsa-miR-377, hsa-miR-451, hsa-miR-486-5p, hsa-miR-766 (fold change $\leq 0.5$ and $p \leq 0.05$ ) whereas only hsa-miR-424-5p was significantly upregulated (fold change $\geq 2$ and $p \leq 0.05$, Table 1). All above-mentioned miRNAs were re-evaluated for further validation. Finally, a significantly elevated hsa-miR-424-5p in SFMCs from AS patients $(8.95 \pm 0.25)$ compared with HCs $(1.31 \pm 0.08, \quad p<0.01) \quad$ (Figure 2a) was notified. As shown in Figure 2b, hsa-miR-377 was significantly decreased in SFMCs from AS patients $(0.01 \pm 0.00)$ compared with $\mathrm{HCs}$ $(0.97 \pm 0.04, p<0.01)$. There were no significant differences of gene expression in other miRNAs among groups (data not shown). Based on target prediction programs (miRTarBase, TargetScan, miRanda, and MiRBase) and published papers, we found highly evidenced candidate target genes (hypoxia-inducible factor $1 \mathrm{~A}$ and Cullin 2 genes for hypoxia and angiogenesis; cyclindependent kinase 6 (CDK6), Cyclin D1 (CCND1), Cyclin E1 (CCNE1), and CCND3 genes for cell cycle) of hsa-miR-424-5p. Meanwhile, protein phosphatase magnesium-dependent 1A (PPM1A) and $\mathrm{p} 21$-activated kinase 1 (PAK1) were predicted as candidate genes of hsa-miR-377 (Table 2).

\section{DISCUSSION}

In the present study, miRNAs in the PBMCs and SFMCs of AS patients were comprehensively

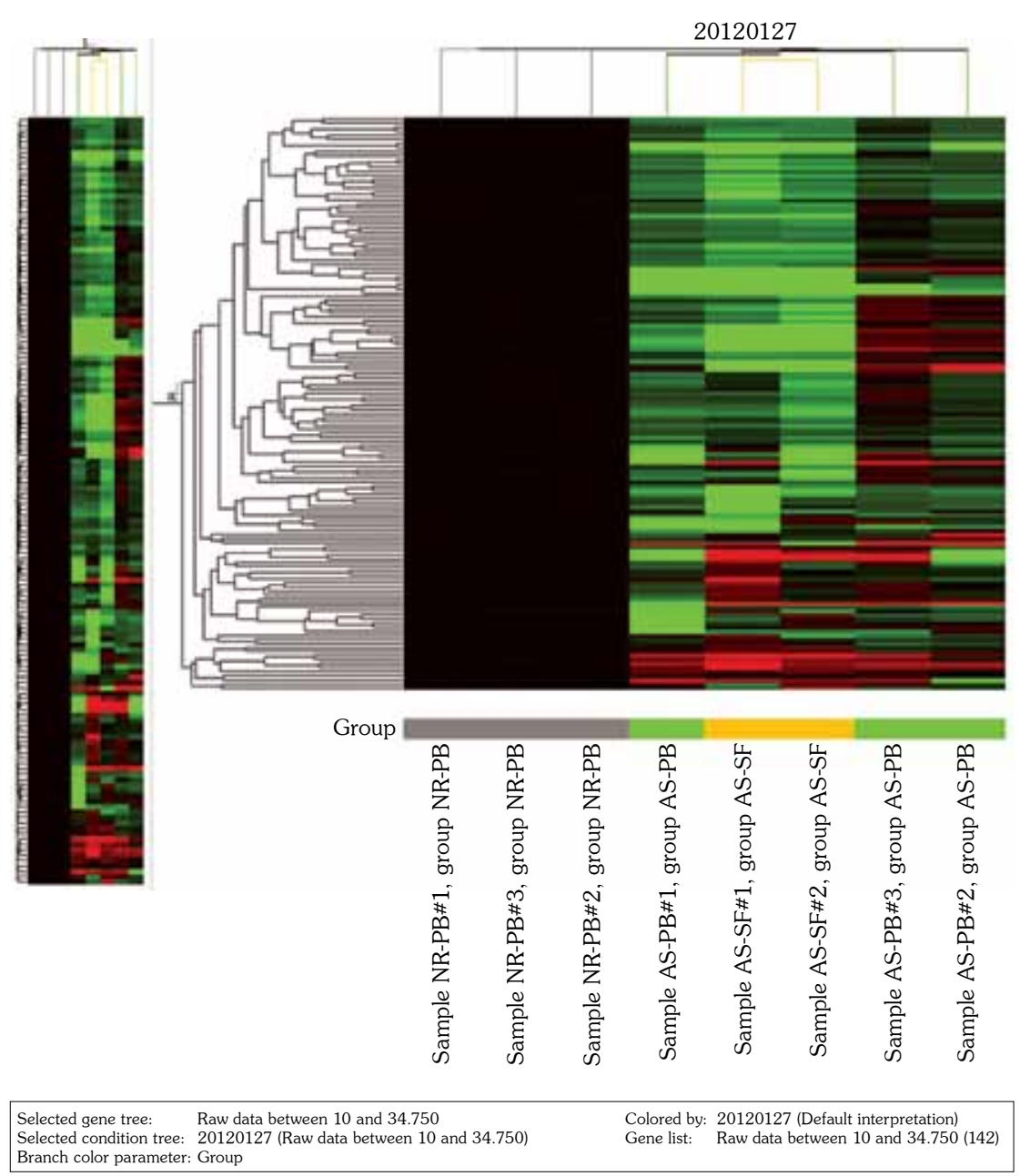

Figure 1. Microarray screening for micro-ribonucleic acids differentially expressed in ankylosing spondylitis. micro-ribonucleic acid expression profiles were monitored across three groups. 
Table 1. Comparison of micro-ribonucleic acid level of peripheral blood mononuclear cells and synovial fluid mononuclear cells in patients with ankylosing spondylitis

\begin{tabular}{|c|c|c|c|c|}
\hline \multirow[b]{2}{*}{ Name of gene } & \multicolumn{2}{|c|}{ PBMC (Fold change) } & \multicolumn{2}{|c|}{ SFMC (Fold change) } \\
\hline & Downregulated & Upregulated & Downregulated & Upregulated \\
\hline hsa-miR-125b & 0.523497 & & 0.01 & \\
\hline hsa-miR-126 & 0.989866 & & 0.01 & \\
\hline hsa-miR-126* & 0.849571 & & 0.01 & \\
\hline hsa-miR-136 & 0.099969 & & 0.01 & \\
\hline hsa-miR-144 & & 1.833485 & 0.01 & \\
\hline hsa-miR-151-3p & & 1.311719 & 0.01 & \\
\hline hsa-miR-199a-5p & 0.994883 & & 0.01 & \\
\hline hsa-miR-29c* & 0.219023 & & 0.01 & \\
\hline hsa-miR-30b & 0.783106 & & 0.445761 & \\
\hline hsa-miR-326 & 0.543503 & & 0.01 & \\
\hline hsa-miR-376a & 0.184359 & & 0.01 & \\
\hline hsa-miR-376c & 0.250179 & & 0.01 & \\
\hline hsa-miR-377 & 0.219750 & & 0.01 & \\
\hline hsa-miR-424-5p & & 2.158072 & & 3.522999 \\
\hline hsa-miR-451 & & 1.921773 & 0.051164 & \\
\hline hsa-miR-486-5p & & 1.091827 & 0.01 & \\
\hline hsa-miR-766 & 0.698829 & & 0.01 & \\
\hline
\end{tabular}

isolated and analyzed by using miRNA microarray analysis. Through this analysis, we discovered significantly high expression of miR-424-5p and lower expression of miR-377 in SFMCs from patients with AS, compared to HCs.

Based on target prediction programs and published papers, miR-424-5p was highly linked to pathways of hypoxia and the cell cycle. Inflammatory arthritis is a chronic progressive autoimmune disease characterized by the proliferation of synovial tissue. The role of hypoxia in the pathogenesis of inflammatory arthritis has been demonstrated in several in vitro and in vivo studies. ${ }^{15-17}$ Hypoxia is a key driving force for angiogenesis and is recognized as an important event in inflammatory arthritis. Several studies have demonstrated hypoxia-inducible factor- $1 \alpha$ expression in synovial tissue. It has been revealed (a)

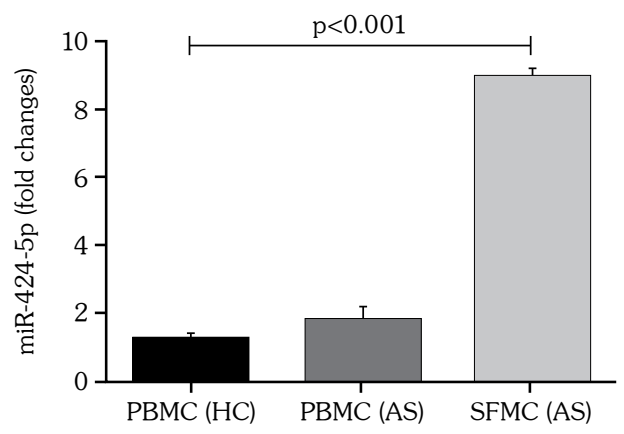

(b)

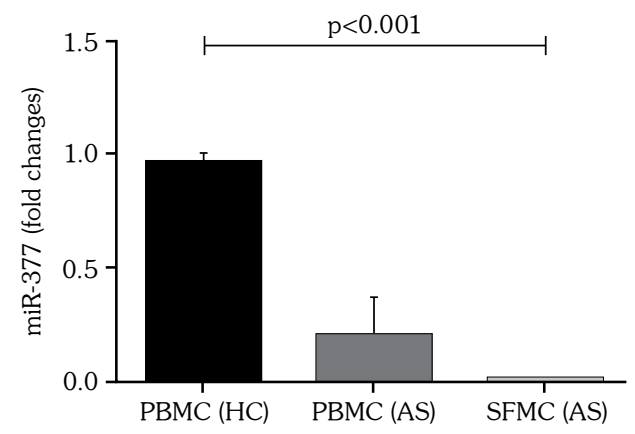

Figure 2. Validation of candidate miRs. Samples from 25 healthy controls (HCs), and paired peripheral blood and synovial fluid from 21 patients with ankylosing spondylitis were obtained. Total ribonucleic acid was collected from each time point. Real-time polymerase chain reaction analysis was performed to determine the expressions of miRs. Values are shown as the mean \pm SEM. The Kruskal-Wallis test was used for the comparison of gene expression among groups. Dunn's test was used post-hoc to compare all pairs of groups. Gene expression was normalized to the U6B snRNA in each sample. PBMC: Peripheral blood mononuclear cell; AS: Ankylosing spondylitis; SFMC: Synovial fluid mononuclear cell; mean \pm SEM: standard error of the mean; snRNA: single small nuclear RNA 
Table 2. Candidate target genes of hsa-miR-424-5p and hsa-miR-377

\begin{tabular}{lccc}
\hline Gene name & Main pathway & Target gene & Accession ID \\
\hline hsa-miR-424-5p & Hypoxia and angiogenesis & HIF1A & MIRT005926 \\
& Cell cycle & CDK6 & MIRT005927 \\
& CCND1 & MIRT000938 \\
& CCNE1 & MIRT000941 \\
& CCND3 & MIRT000937 \\
MAPK pathway & PPM1A & MIRT000990 \\
hsa-miR-377 & PI3K-akt pathway & PAK1 & MIRT000991 \\
HIF1A: Hypoxia-inducible factor 1A; CUL2: Cullin 2; CDK: Cyclin-dependent kinase; CCND1: Cyclin \\
$\begin{array}{l}\text { D1; CCNE1: Cyclin E1; CCND3: Cyclin D3; MAPK: Mitogen-activated protein kinase; PPM1A: Protein } \\
\text { phosphatase magnesium-dependent 1A; PI3K: Phosphatidylinositol 3'-kinase; PAK1: p21-activated } \\
\text { kinase 1. }\end{array}$ \\
\hline
\end{tabular}

in synovial cell cultures, that hypoxia induces key angiogenic growth factors, chemokines, and matrix metalloproteinases. ${ }^{18,19}$ Various stresses, including hypoxia, regulate the expression and function of miRNA. ${ }^{20}$ For example, a subset of miRNAs induced by hypoxia contributes to the regulation of the broad spectrum of genes regulated by hypoxia. It was reported that miR-424-5p is a critical mediator of oxygen-dependent changes in endothelial cells and is physiologically upreg ulated in tissues undergoing vascular remodelin $\mathrm{g}$ and angiogenesis. ${ }^{21}$ Cyclin D1, the product of CCND1 gene, is an important regulator of G1 to $\mathrm{S}$ phase progression in many different cell types. Cyclin D1, together with its binding partners CDK4 and CDK6, forms active complexes that promote cell cycle progression by phosphorylating and inactivating the retinoblastoma protein. ${ }^{22}$ According to our data, miR-424-5p might inhibit cell proliferation, arrest cell cycle progression, and induce cell apoptosis in AS.

Recently, it was published that PPM1A was highly expressed in AS synovial tissue. Moreover, PPM1A detecting antibodies were correlated positively with Bath Ankylosing Spondylitis Disease Activity Index score. ${ }^{23}$ Given the result from our data, decreased expression of miR-377 in SFMCs might contribute to increase levels of PPM1A in AS synovium. The expression of PAK1 was increased in rheumatoid arthritis fibroblastlike synoviocytes fibroblast-like synoviocytes. PAK1 plays an important role in regulating the migration, invasion and production and activity of matrix metalloproteinases in fibroblastlike synoviocytes, which is mediated by the c-Jun N-terminal kinase pathway. ${ }^{24}$ This might suggest that hsa-miR-377 has a role on PAK1 expression in arthritis of AS, although there is no report of PAK1 in AS.

In conclusion, miR-424-5p was increased and miR-377 was decreased in SFMCs from patients with AS. These two miRs might have functional roles in patients with arthritis via different pathways. However, further research is necessary to ascertain whether miR-424-5p and miR-377 can truly have functional roles in AS patients with peripheral arthritis. Due to the small sample size, further validation of other candidate miRNAs from this microarray assay should be carried out. Nevertheless, data from this microarray may provide important information for research on miRNA in AS.

\section{Declaration of conflicting interests}

The authors declared no conflicts of interest with respect to the authorship and/or publication of this article.

\section{Funding}

This study was supported by grants from the National Research Foundation of Korea (NRF) funded by the Ministry of Education, Science, and Technology (grant no. 2011-0008867), by the Chonnam National University Hospital Research Institute of Clinical Medicine (grant no. CRI 11074-21).

\section{REFERENCES}

1. Khan MA, van der Linden SM. A wider spectrum of spondyloarthropathies. Semin Arthritis Rheum 1990;20:107-13.

2. Kim TJ, Lee S, Joo KB, Park DJ, Park YW, Lee SS, et al. The presence of peripheral arthritis delays spinal 
radiographic progression in ankylosing spondylitis: Observation Study of the Korean Spondyloarthropathy Registry. Rheumatology (Oxford) 2014;53:1404-8.

3. Kim TJ, Kim TH. Clinical spectrum of ankylosing spondylitis in Korea. Joint Bone Spine 2010;77:235-40.

4. Heuft-Dorenbosch L, van Tubergen A, Spoorenberg $\mathrm{A}$, Landewé $\mathrm{R}$, Dougados $\mathrm{M}$, Mielants $\mathrm{H}$, et al. The influence of peripheral arthritis on disease activity in ankylosing spondylitis patients as measured with the Bath Ankylosing Spondylitis Disease Activity Index. Arthritis Rheum 2004;51:154-9.

5. Bartel DP. MicroRNAs: genomics, biogenesis, mechanism, and function. Cell 2004;116:281-97.

6. O'Connell RM, Rao DS, Chaudhuri AA, Baltimore D. Physiological and pathological roles for microRNAs in the immune system. Nat Rev Immunol 2010;10:111-22.

7. Pillai RS, Bhattacharyya SN, Filipowicz W. Repression of protein synthesis by miRNAs: how many mechanisms? Trends Cell Biol 2007;17:118-26.

8. Jin HM, Kim TJ, Choi JH, Kim MJ, Cho YN, Nam KI, et al. MicroRNA-155 as a proinflammatory regulator via SHIP-1 down-regulation in acute gouty arthritis. Arthritis Res Ther 2014;16:88.

9. Rodriguez A, Vigorito E, Clare S, Warren MV, Couttet $\mathrm{P}$, Soond DR, et al. Rodriguez A, Vigorito E, Clare $\mathrm{S}$, Warren MV, Couttet P, Soond DR, et al. Science 2007;316:608-11.

10. Xiao C, Srinivasan L, Calado DP, Patterson HC, Zhang B, Wang J, et al. Lymphoproliferative disease and autoimmunity in mice with increased miR17-92 expression in lymphocytes. Nat Immunol 2008;9:405-14.

11. Murata K, Yoshitomi $\mathrm{H}$, Tanida S, Ishikawa $\mathrm{M}$, Nishitani $\mathrm{K}$, Ito $\mathrm{H}$, et al. Plasma and synovial fluid microRNAs as potential biomarkers of rheumatoid arthritis and osteoarthritis. Arthritis Res Ther 2010;12:86.

12. Huang J, Song G, Yin Z, Luo X, Ye Z. Elevated miR-29a expression is not correlated with disease activity index in PBMCs of patients with ankylosing spondylitis. Mod Rheumatol 2014;24:331-4.

13. Lai NS, Yu HC, Chen HC, Yu CL, Huang HB, Lu MC. Aberrant expression of microRNAs in $T$ cells from patients with ankylosing spondylitis contributes to the immunopathogenesis. Clin Exp Immunol 2013;173:47-57.

14. Huang CH, Wei JC, Chang WC, Chiou SY, Chou
$\mathrm{CH}$, Lin YJ, et al. Higher expression of whole blood microRNA-21 in patients with ankylosing spondylitis associated with programmed cell death 4 mRNA expression and collagen cross-linked C-telopeptide concentration. J Rheumatol 2014;41:1104-11.

15. Konisti S, Kiriakidis S, Paleolog EM. Hypoxia--a key regulator of angiogenesis and inflammation in rheumatoid arthritis. Nat Rev Rheumatol 2012;8:153-62.

16. Del Rey MJ, Izquierdo E, Usategui A, Gonzalo E, Blanco FJ, Acquadro F, et al. The transcriptional response of normal and rheumatoid arthritis synovial fibroblasts to hypoxia. Arthritis Rheum 2010;62:3584-94.

17. Brouwer E, Gouw AS, Posthumus MD, van Leeuwen MA, Boerboom AL, Bijzet $\mathrm{J}$, et al. Hypoxia inducible factor-1-alpha (HIF-1alpha) is related to both angiogenesis and inflammation in rheumatoid arthritis. Clin Exp Rheumatol 2009;27:945-51.

18. Akhavani MA, Madden L, Buysschaert I, Sivakumar B, Kang N, Paleolog EM. Hypoxia upregulates angiogenesis and synovial cell migration in rheumatoid arthritis. Arthritis Res Ther 2009;11:64.

19. Muz B, Khan MN, Kiriakidis S, Paleolog EM. Hypoxia. The role of hypoxia and HIF-dependent signalling events in rheumatoid arthritis. Arthritis Res Ther 2009;11:201.

20. Kulshreshtha R, Ferracin M, Wojcik SE, Garzon $\mathrm{R}$, Alder $\mathrm{H}$, Agosto-Perez FJ, et al. A microRNA signature of hypoxia. Mol Cell Biol 2007;27:1859-67.

21. Ghosh G, Subramanian IV, Adhikari N, Zhang X, Joshi HP, Basi D, et al. Hypoxia-induced microRNA-424 expression in human endothelial cells regulates HIF- $\alpha$ isoforms and promotes angiogenesis. $\mathrm{J}$ Clin Invest 2010;120:4141-54.

22. Alao JP. The regulation of cyclin D1 degradation: roles in cancer development and the potential for therapeutic invention. Mol Cancer 2007;6:24.

23. Kim YG, Sohn DH, Zhao X, Sokolove J, Lindstrom TM, Yoo B, et al. Role of protein phosphatase magnesium-dependent $1 \mathrm{~A}$ and antiprotein phosphatase magnesium-dependent $1 \mathrm{~A}$ autoantibodies in ankylosing spondylitis. Arthritis Rheumatol 2014;66:2793-803.

24. Fu D, Yang Y, Xiao Y, Lin H, Ye Y, Zhan Z, et al. Role of $\mathrm{p} 21$-activated kinase 1 in regulating the migration and invasion of fibroblast-like synoviocytes from rheumatoid arthritis patients. Rheumatology (Oxford) 2012;51:1170-80. 